\title{
High-energy laser detection through thermoelectric generators
}

\author{
Joseph Merkel, ${ }^{\text {a }}$ Steven Yee, ${ }^{\mathrm{a}}$ Charles Nelson, ${ }^{\mathrm{a}}$ Brian Jenkins, ${ }^{\mathrm{a}}$ \\ Hatem ElBidweihy, ${ }^{\text {a }}$ Peter Joyce, ${ }^{\text {b }}$ Cody Brownell, ${ }^{\text {b }}$ \\ and Deborah Mechtel ${ }^{\mathrm{a}, *}$ \\ ${ }^{a}$ U.S. Naval Academy, Electrical and Computer Engineering Department, Annapolis, \\ Maryland, United States \\ ${ }^{b}$ U.S. Naval Academy, Mechanical Engineering Department, Annapolis, Maryland, United States
}

\begin{abstract}
Detection of high-energy laser strikes is key to the survivability of military assets in future warfare. The introduction of laser weapon systems demands the capability to quickly detect these strikes without disrupting the stealth capability of military craft with active sensing technologies. We explored the use of thermoelectric generators (TEGs) as self-powered passive sensors to detect such strikes. Experiments were conducted using lasers of various power ratings, wavelengths, and beam sizes to strike $2 \times 2 \mathrm{~cm}^{2}$ commercially available TEGs arranged in different configurations. Open-circuit voltage and short-circuit current responses of TEGs struck with 808-, 1070-, and 1980-nm lasers at irradiance levels between 8.5 and $509.3 \mathrm{~W} / \mathrm{cm}^{2}$ and spot sizes between 2 and $8 \mathrm{~mm}$ are compared. TEG surface temperatures indicate that the sensor can survive temperatures nearing $400^{\circ} \mathrm{C}$. TEG open-circuit voltage magnitudes correlate more strongly with net incident laser power than with specific irradiance levels, and linearity is limited by Seebeck coefficient variation with temperature. Open-circuit voltage responses are characterized by $10 \%$ to $90 \%$ rise times of $\sim 2$ to $10 \mathrm{~s}$ despite surface temperatures not reaching equilibrium. With open-circuit voltage as the sensing parameter, detection thresholds three times above the standard deviation noise level can be exceeded within $300 \mathrm{~ms}$ of the start of a laser strike with irradiance levels of $\sim 200 \mathrm{~W} / \mathrm{cm}^{2}$. Potential harvested power levels as high as $16 \mathrm{~mW}$ are estimated based on measured electrical responses. A multiphysics finite-element model corresponding to the experiments was developed to further optimization of a lightweight, lowprofile TEG sensor for detection of high-energy laser strikes. (C) The Authors. Published by SPIE under a Creative Commons Attribution 4.0 Unported License. Distribution or reproduction of this work in whole or in part requires full attribution of the original publication, including its DOI. [DOI: 10.1117/1.OE .59.11.117105]
\end{abstract}

Keywords: thermoelectric generator; high-energy laser detection.

Paper 20200825 received Jul. 9, 2020; accepted for publication Sep. 16, 2020; published online Nov. 19, 2020.

\section{Introduction}

Laser weapon systems are difficult to detect because they are both silent and invisible. The only methods of detection are to receive the laser radiation into an optical system or to detect an increase in temperature on the target. Infrared (IR) cameras, which fall into the former category, are sensitive to a wide range of wavelengths, but must be viewing the target to observe a strike. For military applications, assets such as tanks, aircraft, ships, and drones need to operate independently, which means an extra asset deployed solely for detecting laser radiation via an IR camera is less practical. Companies such as Boeing and Carl Zeiss have patented other technologies that include an externally mounted device on the asset to receive laser radiation. ${ }^{1,2}$ Both technologies are bulky and can be mounted to a limited set of platforms. In addition to having poor aerodynamic geometry, these devices disrupt the stealth ability of their asset by protruding far outside its skin, allowing an adversary's radar to easily detect the asset. Embedded photoconductive sensing techniques have also been investigated but required direct

*Address all correspondence to Deborah Mechtel, E-mail: mechtel@usna.edu 
exposure to laser photons and have limited potential to scale to cover large areas due to the physically large radio-frequency readout instruments required to read continuously active signals. ${ }^{3,4}$ Among temperature-sensing technologies, optical fiber sensors, including fiber Bragg gratings, embedded in composite structures have been investigated for high-energy laser strike detection applications. ${ }^{5-7}$ Fiber optic sensors are limited by brittleness if manufactured using glass ${ }^{8}$ or material degradation at temperatures exceeding $70^{\circ} \mathrm{C}$ if manufactured using polymethyl methacrylate (PMMA), ${ }^{9}$ which would not survive a high laser intensity environment. Additionally, distributed fiber optic sensors require active interrogation and are sensitive to strain in addition to temperature, ${ }^{10}$ requiring significant processing effort to isolate the two effects.

Thermoelectric generators (TEGs) employed as high-energy laser strike sensors offer advantages compared to these other technologies because TEGs operate passively, are durable against high temperatures, and can be constructed in thin and flexible forms for integration onto structural platforms. Moreover, because TEGs are sensitive to temperature and relatively insensitive to other phenomena such as strain, they are directly related to the damage mechanism expected from a high-energy laser strike. A TEG responds to a temperature gradient according to the Seebeck effect. The Seebeck effect, which is reciprocal to the more commonly known Peltier effect, states that if a temperature gradient exists across dissimilar materials such as $p$ - and $n$-type semiconductors, the junction between the materials will produce a voltage difference. With a larger temperature gradient, the generated voltage increases. The theory of operation for a TEG as a laser strike sensor is that a high-energy laser strike will rapidly heat the exterior irradiated surface of the TEG, creating a temperature gradient through the TEG thickness and a resultant rise in the open-circuit voltage of the TEG. A certain voltage threshold could be used to indicate a high-energy laser strike. TEGs are not only passive but also essentially powered by the laser strikes intended to be detected, and therefore, do not need to be powered by the target seeking to detect the strike. The TEGs used in this project, CUI Device's CP60240, are manufactured on a $96 \%$ aluminum oxide substrate, which is physically very durable and has a high melting point of greater than $2000^{\circ} \mathrm{C} .{ }^{11,12}$ The dimensions of the tested models are $2 \mathrm{~cm} \times 2 \mathrm{~cm} \times 4 \mathrm{~mm}$, but thinner models have been manufactured. ${ }^{13}$ TEGs have been manufactured with micrometer scale thicknesses ${ }^{14}$ and in flexible forms. ${ }^{15}$

In this paper, we evaluate the ability of commercial-off-the-shelf TEGs to detect laser strikes. ${ }^{16}$ Using lasers of various powers and spot sizes, we measure variation in TEG open-circuit voltage, short-circuit current, and surface temperatures as a function of laser power and average irradiance levels. While higher incident laser power is expected to produce higher open-circuit voltages in the TEG due to more rapid heating, we also assess the effect of specific irradiance at a fixed power level by varying the spot size of the incident laser. Higher irradiance is also expected to result in more rapid heating of the TEG, but a significant thermal gradient through the TEG thickness will be present over fewer thermoelectric couples when the spot size is small. Tests were conducted to observe the failure point of the TEGs when exposed to increasing temperatures levels due to increasingly powerful laser strikes. Additionally, because the absorption of light by a material varies with the wavelength of the light, several different wavelength lasers were used to determine the wavelength dependence of the TEG thermal and electrical response. We further develop a multiphysics finite-element model corresponding to the experiments conducted to determine the effect of material parameters on predicted TEG sensor performance. These results will inform material selections in designs for functional high-energy laser strike sensors based on TEGs.

\section{Methods}

\subsection{Experimental Methods}

The basic experiment was striking a single TEG with a laser centered on the TEG surface. An example is shown in Fig. 1(a). Variations in laser power and wavelength were obtained via a LaserMotive $808 \mathrm{~nm}$ 40-W power over fiber laser (PN: 210-6443) ranging from 15 to $40 \mathrm{~W}$, an IPG Photonics YLR-100-SM-CS 1070-nm fiber laser ranging from 25 to $100 \mathrm{~W}$, and an IPG Photonics TLR-50-1980-nm fiber laser ranging from 25 to $50 \mathrm{~W}$. A ThorLabs S314C thermal power sensor head was used to verify the claimed net output power of each laser before 

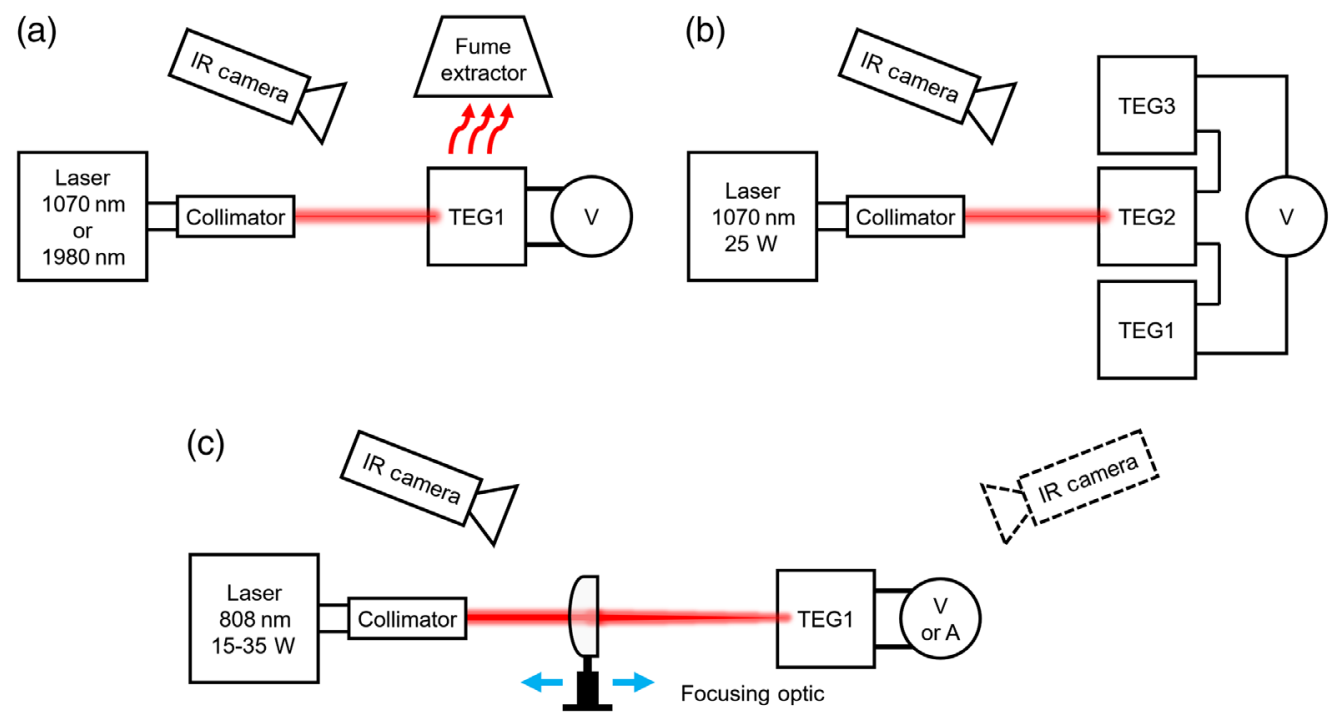

Fig. 1 Schematic representations of experiments performed. (a) Single TEG irradiation using a 1070- or 1980-nm laser with varying power levels, (b) irradiation of TEGs in series configuration, and (c) irradiation of TEG with an 808-nm laser with varying spot size via a repositionable lens.

commencing experiments. For spot size diameters of $\sim 8 \mathrm{~mm}$, irradiance levels ranged from 8.5 to $198.9 \mathrm{~W} / \mathrm{cm}^{2}$. Real-time temperature monitoring was conducted via IR cameras imaging either the front (irradiated) or rear surface of the TEG. A forward-looking infrared system (FLIR) A615 IR camera was used to record the temperatures on the front of the TEG for the 1070- and 1980-nm laser strikes, and an FLIR A325sc IR camera was used to record the temperatures on the front and back of the TEG for the 808-nm laser strikes. The difference in camera type for each experiment was based solely on availability at the time of the experiments. Opencircuit voltage responses of the TEG were measured using a DSO9104A Digital Storage Oscilloscope or a Wavesurfer 104MXs 1GHz 5GS/s Oscilloscope. For the 808-nm laser strikes, short-circuit current responses were also measured using a digital multimeter. Due to changes in the testing venue, the 808-nm experiments were conducted on TEGs supported by an adjustable lens mount, leaving the rear of the TEG exposed, while the 1070- and 1980-nm experiments were conducted on TEGs supported by a three-dimensional (3-D)-printed mounting bracket that covered the rear surface of the TEG. Additionally, a fume extractor was situated above the TEG for the 1070- and 1980-nm experiments in case the TEG produced any smoke, but no fume extractor was available for the 808 -nm experiments.

In addition to strikes on a single TEG, experiments were conducted to assess the behavior of TEGs in a system, with concern for system performance after the failure of a device. Three TEGs were arranged in series with the oscilloscope connected across the entire line of TEGs, as seen in Fig. 1(b). The laser struck the middle TEG (TEG2) and the voltage response was recorded. TEG3 was then replaced with a broken TEG, one that had failed after a previous high-power laser strike, and the experiment was repeated. To further test the effect of a broken TEG in the series arrangement, TEG3 was returned, and TEG1 was replaced with another broken TEG, and the experiment was repeated.

To assess the effect of specific irradiance levels on TEG response, the 808-nm laser was directed at a single TEG with a planoconvex focusing optic (ThorLabs uncoated N-BK7, $300-\mathrm{mm}$ focal length) inserted in front of the collimator and adjusted to vary the specific irradiance at the TEG as shown in Fig. 1(c). Spot size diameters of 2, 5, and $8 \mathrm{~mm}$ were used at $25 \mathrm{~W}$, with irradiances of $795.8,127.3$, and $49.7 \mathrm{~W} / \mathrm{cm}^{2}$, respectively. Then, a $15-\mathrm{mm}$ spot size was used at 15,25 , and $35 \mathrm{~W}$, with irradiances of $2.12,3.54$, and $4.95 \mathrm{~W} / \mathrm{cm}^{2}$, respectively. The IR camera was used to view the front of the TEG and then repositioned to view the back of the TEG for these trials.

Additional experiments were conducted where a planoconvex focusing optic (ThorLabs uncoated N-BK7, 200-mm focal length) was inserted in front of the 1070-nm laser to focus 
the spot size on the TEG to $5 \mathrm{~mm}$. These tests were used to observe the behavior of the TEG when exposed to extreme irradiances. These trials used the same nominal laser power settings of $25,50,75$, and $100 \mathrm{~W}$ as were used in the experiments conducted with the same 1070-nm laser without a focusing optic. However, the nominal laser power settings were not adjusted to account for transmission loss through the focusing optic such that the actual power on the TEG was equivalent to those trials that did not use an optic. Based on an estimated $92 \%$ transmission for ThorLabs N-BK7 uncoated lenses at the 1070-nm wavelength, we estimate irradiances at the TEG of $117.1,234.2,351.4$, and $468.6 \mathrm{~W} / \mathrm{cm}^{2}$ for the nominal 25-, 50-, 75-, and 100-W trials focused with the optic, respectively. However, since these irradiances were not measured, we do not compare the responses of the TEGs in these trials quantitatively against those trials conducted without a focusing optic on the basis of power.

\subsection{Simulation Methods}

A finite-element multiphysics model approximately corresponding to the experiments conducted was developed using COMSOL Multiphysics ${ }^{\circledR}$ to compare simulation responses to those obtained experimentally for parameter estimation and to evaluate the relevance of certain parameters to the thermal and electrical response of the tested TEG. The model was developed using physical dimensions measured from a deconstructed part, built-in COMSOL material library parameters for bismuth telluride and copper, and published material parameters for $96 \%$ aluminum oxide. ${ }^{17}$ The heat transfer in solids and electric currents modules were utilized, including the multiphysics module for the thermoelectric effect. Because the thermal experimental conditions to which the simulations would be compared did not reach steady-state equilibrium, timedependent transient simulation studies were conducted instead of stationary steady-state studies. Ambient temperature was set to $20^{\circ} \mathrm{C}$ or $23^{\circ} \mathrm{C}$ to match the corresponding experiments. A summary of relevant simulation parameters is included in Appendix A.

Laser input was modeled as a "deposited beam power" with a Gaussian profile of parameterized standard deviation $\sigma_{\text {spot }}$ centered on the exterior surface of the top aluminum oxide domain. The Gaussian profile is not confirmed experimentally as the beam profiles were not characterized. Nominal integrated input heating power (laser power) was modeled as a smoothed step function from zero to a parameterized maximum value $P_{\text {laser }}$ with a 100 -ms-duration transition zone centered at the 50-ms mark with two continuous derivatives applied in the smoothing function. The actual power modeled as absorbed at the aluminum oxide surface was further modified by the wavelength-dependent emissivity $\varepsilon(\lambda)$. This emissivity is a key parameter that is estimated through the simulation process and was expected to be significantly $<0.4$ at the nearIR wavelengths tested based on published literature. ${ }^{18}$ Some small level of transmission to the back alumina surface was also considered using deposited beam power while accounting for absorption and potential reflection off the front aluminum oxide surface. For simplicity, the central exterior region of the TEG model neglected the silicone rubber sealant and assumed thermally insulating boundary conditions at this interface. Convective heat transfer conditions with parameterized coefficients $h_{\text {front }}$ and $h_{\text {back }}$ were applied to the front and back surfaces of the aluminum oxide domains, respectively.

Electrical aspects of the simulation model included copper ohmic contacts between 25 thermoelectric pairs. Due to local inability to directly test the Seebeck coefficients of the materials in the commercial TEGs tested, the COMSOL built-in material library parameters for bismuth telluride were used, including COMSOL's built-in function for the Seebeck coefficient $S(T)$ as a function of temperature. Outside of the defined temperature range of 200 to $350 \mathrm{~K}$, the Seebeck coefficient was modeled as a constant equal to the nearest value indicated in Table 1 . A scaling coefficient $\beta$ was also applied to the Seebeck coefficient function as a control variable to tune the simulation results to the experimental results. Variation in effective Seebeck coefficients can be wide even among nominally the same materials based on processing conditions and with variation due to temperature. ${ }^{19}$ For simplicity, the $n$ - and $p$-type materials were assumed to have exactly opposite Seebeck coefficients. The terminal ohmic contacts were designated as either ground or a terminal by which to measure the simulated open-circuit voltage response of the TEG.

The simulation approach focused on estimating the wavelength-dependent emissivity of aluminum oxide and the convective heat transfer coefficients by comparing the simulated surface 
Table 1 Summary of open-circuit voltage $\left(V_{\mathrm{OC}}\right)$ and recorded average surface temperatures on the front of the TEG for selected experiments.

\begin{tabular}{|c|c|c|c|c|c|c|c|c|c|}
\hline \multirow{2}{*}{$\frac{\lambda}{\text { Power (W) }}$} & \multicolumn{3}{|c|}{$808 \mathrm{~nm}$} & \multicolumn{4}{|c|}{$1070 \mathrm{~nm}$} & \multicolumn{2}{|c|}{$1980 \mathrm{~nm}$} \\
\hline & & 25 & & 25 & 50 & 75 & 100 & 25 & 50 \\
\hline Irradiance $\left(\mathrm{W} / \mathrm{cm}^{2}\right)$ & 49.7 & 127.3 & 795.8 & 49.7 & 99.5 & 149.2 & 198.9 & 49.7 & 99.5 \\
\hline$V_{\mathrm{OC}}$ at $t=4 \mathrm{~s}(\mathrm{mV})$ & 152 & 150 & 136 & 113 & 246 & 397 & 493 & 280 & 550 \\
\hline Average temperature at $t=4 \mathrm{~s}\left({ }^{\circ} \mathrm{C}\right)$ & 41 & 38 & $n / r^{\mathrm{a}}$ & 31 & 42 & 52 & 69 & 53 & 77 \\
\hline
\end{tabular}

aTemperature data for the 808-nm, 2-mm spot size strike was not recorded.

temperatures obtained from sweeps of these parameters to those observed experimentally using IR cameras. Once the simulated surface temperature transient response was reasonably close to the experimental results, the Seebeck coefficient scaling parameter $\beta$ was adjusted as necessary to match the simulated voltage responses to the experimental open-circuit voltage responses. Additional simulation studies included the response of the TEG to changes in integrated laser input power by sweeping parameter $P_{\text {laser }}$ and to the spot size of the laser by sweeping parameter $\sigma_{\text {spot }}$.

\section{Results}

\subsection{Experimental Results}

As predicted, the voltage of the TEG increased as the temperature at its surface increased. Figure 2 shows that each increase in power increases the TEG voltage response to the 1070-nm laser with an 8-mm spot size, until voltage responses saturate as they approach $800 \mathrm{mV}$. Two breakdowns can be observed in this plot. The first was the $100-\mathrm{W}$ trial's clear drop to $0 \mathrm{~V}$ at the 18 -s mark. The other was the $75-\mathrm{W}$ trial's decrease in open-circuit voltage starting at the 14-s mark. In the $75-\mathrm{W}$ breakdown, the voltage achieved $700 \mathrm{mV}$ before steadily declining. After both trials, the TEG could no longer produce a voltage in response to a temperature gradient. The 1980-nm laser experiment at $50 \mathrm{~W}$ with an 8-mm-diameter spot size also experienced a breakdown after reaching an open-circuit voltage of $800 \mathrm{mV}$ under laser irradiation. In Fig. 3(a) at the 12-s mark, the voltage begins to decline, which was another indication of a breakdown. While this declining open-circuit voltage was not always an indication of a

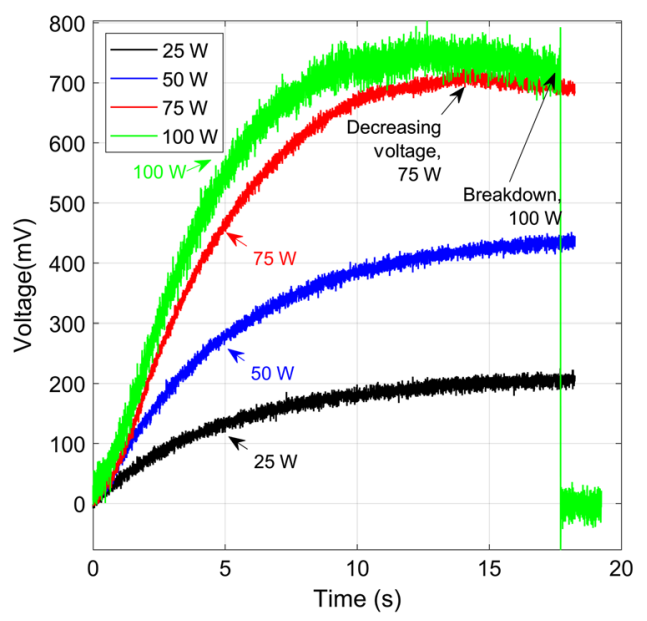

Fig. 2 Open-circuit voltage responses of TEG to 1070-nm laser strikes of varying net power levels and a fixed 8-mm-diameter spot size. Increasing incident power levels increase the output voltage but saturate above $75 \mathrm{~W}$. The voltage response of the TEG to the $100-\mathrm{W}$ strike indicates a material open-circuit failure of the TEG at around the 18-s mark. 

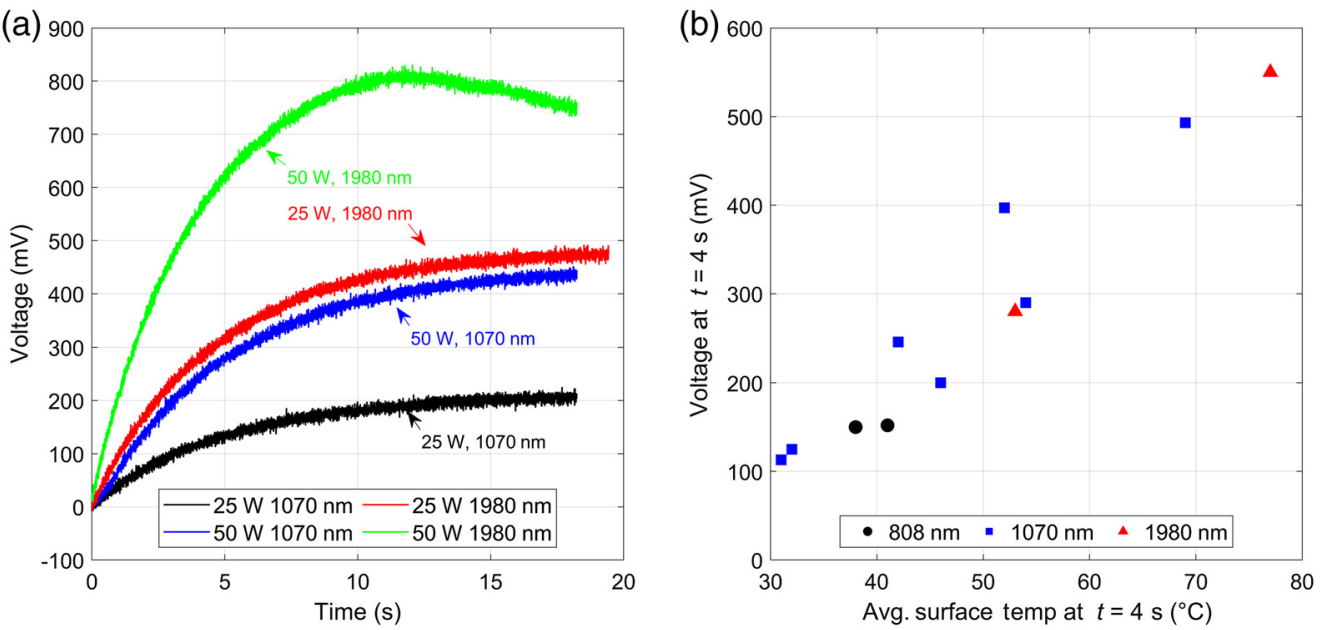

Fig. 3 (a) Comparison of open-circuit voltage responses of a TEG to 1070- and 1980-nm laser strikes at 25- and 50-W net power levels with a spot size diameter of $8 \mathrm{~mm}$. The 1980-nm wavelength laser strikes resulted in significantly larger temperatures and open-circuit voltage responses compared to the 1070-nm wavelength strikes, pointing to variation in the emissivity of the aluminum oxide substrate between the two wavelengths. (b) Comparison of measured open-circuit voltage responses to measured average surface temperature $4 \mathrm{~s}$ after the start of laser strikes of various wavelengths and intensities.

breakdown, in these trials, the TEG was rendered broken afterward, behaving as an open circuit in any subsequent trial.

The wavelength of the laser had a significant effect on the open-circuit voltage response of the TEG. Figure 3(a) compares the 1070- and 1980-nm lasers at 25 and $50 \mathrm{~W}$. The 1980-nm laser at $25 \mathrm{~W}$ returned a higher voltage from the TEG than the $1070-\mathrm{nm} 50-\mathrm{W}$ laser pointing to variations in the emissivity of the aluminum oxide substrate between the two wavelengths. And, as mentioned previously, the 1980-nm 50-W laser caused a breakdown, while the TEG survived the 1070-nm laser at that same power. Only the 1070 and $1980 \mathrm{~nm}$ wavelengths are used to compare the effects of wavelength because only these trials utilized a fumed extractor, had no lens to cause transmission loss between the collimator and the TEG, and covered the back of the TEG with a support bracket. The 808-nm experiments required a lens to focus the beam to a comparable size, left the back of the TEG exposed, and did not use a fume extractor above the TEG. The 808-nm experiment data were instead used to analyze the effects of irradiance and observe heat transfer through the TEG.

In Fig. 3(b), the observed average surface temperature on the irradiated side of the TEG and the open-circuit voltage response of the TEG are compared for experiments spanning different wavelengths and net incident laser powers. For each experiment, the average surface temperature and the open-circuit voltage are measured $4 \mathrm{~s}$ after the start of the laser strike. Linear correlation is observed between the average surface temperatures on the TEG and the open-circuit voltages measured at the 4-s mark across a range of laser wavelengths, power densities, and specific irradiances. In each experiment, the laser continues to irradiate the TEG at the 4-s mark. The linearly correlated relationship between temperature and open-circuit voltage does not hold when compared after a prolonged laser strike. As observed in Fig. 4, which is generally representative of the experiments, surface temperatures continued to rise even as open-circuit voltages reached a steady state.

The average temperature over the front surface of the TEG and the temperature at the hottest point on the TEG were measured using the FLIR tools software that collected data over the entire front square of the target. These data were collected for the 1980- and 1070-nm experiments. For the 5-mm-diameter spot size strikes, the difference between the maximum and average temperature over the surface of the TEG was greater than that of the 8-mm-diameter spot. The exact maximum temperature could not be determined because the IR camera can only detect up to $2090^{\circ} \mathrm{C}$. A summary of open-circuit voltage and recorded average surface temperatures on the front of the TEG for select experiments is shown in Table 1. 

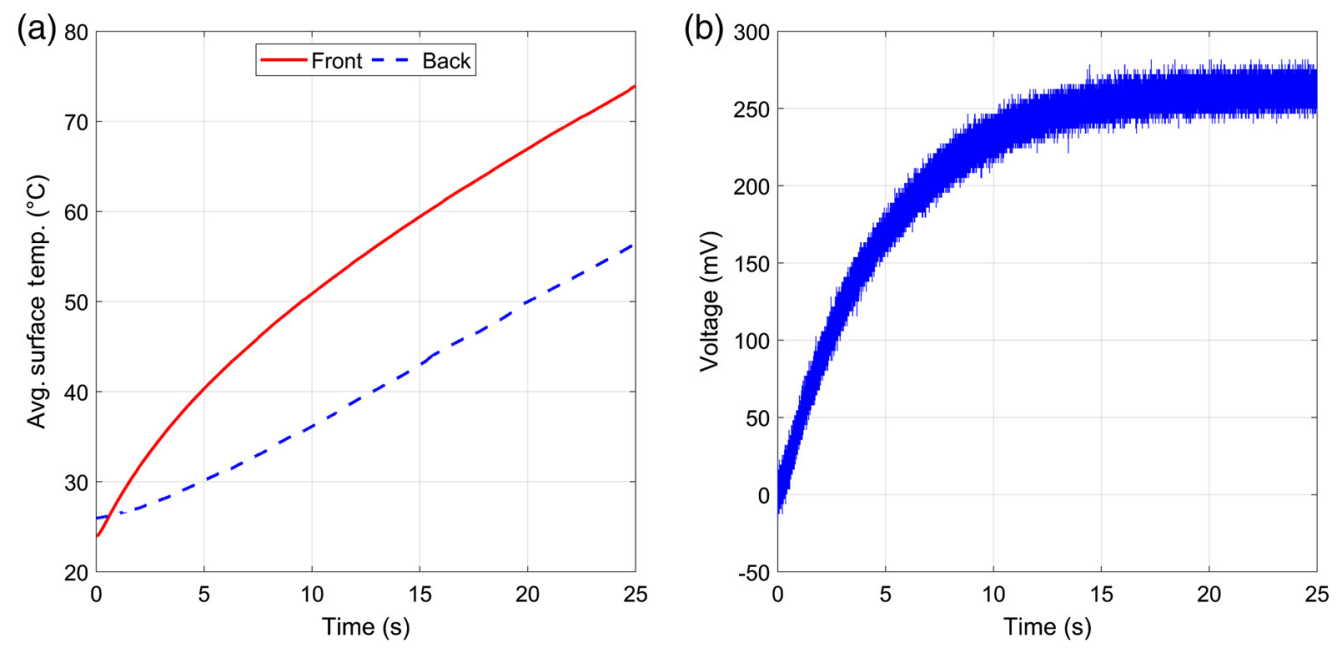

Fig. 4 (a) Observed average temperatures on the laser-irradiated (front) and nonlaser-irradiated (back) side of a TEG during a strike by a $25-\mathrm{W}$ 808-nm laser with a 5-mm spot size. (b) The corresponding output voltage response. Although the average surface temperatures continue to increase, the open-circuit voltage response reaches a maximum.

The heat transfer through the TEG can be observed using the temperature data from the front and back of the TEG during the 808-nm experiments, as seen in Fig. 4. Over the duration of the strike, the temperature on the front of the TEG consistently increased, with the back temperature also rising at nearly the same rate. However, the voltage reached a steady state even as the temperature continued to increase. Between 15 and $25 \mathrm{~s}$, the temperature on the front and back of the TEG increased by about $14^{\circ} \mathrm{C}$ while the TEG voltage remained relatively constant.

Specific irradiance was the least influential parameter on the voltage response from the TEG compared to wavelength and nominal net incident power. While larger spot sizes yielded higher voltages, the differences were minute. The steady voltages resulting from 2-, 5-, and 8-mm lasers of the same power varied by less than $15 \mathrm{mV}$, as shown in Fig. 5. This was noteworthy, as a higher specific irradiance at a fixed net power level slightly reduced the voltage response but greatly increased the likelihood of a breakdown because it was subject to extreme temperatures in a concentrated area.

To assess the noise of the open-circuit voltage response, the voltage response for each trial was high-pass filtered with a cutoff frequency of $2 \mathrm{~Hz}$. The bandwidth of the signal was calculated from this cutoff up to the Nyquist frequency of $5 \mathrm{kHz}$ to isolate the noise of the signal. Most

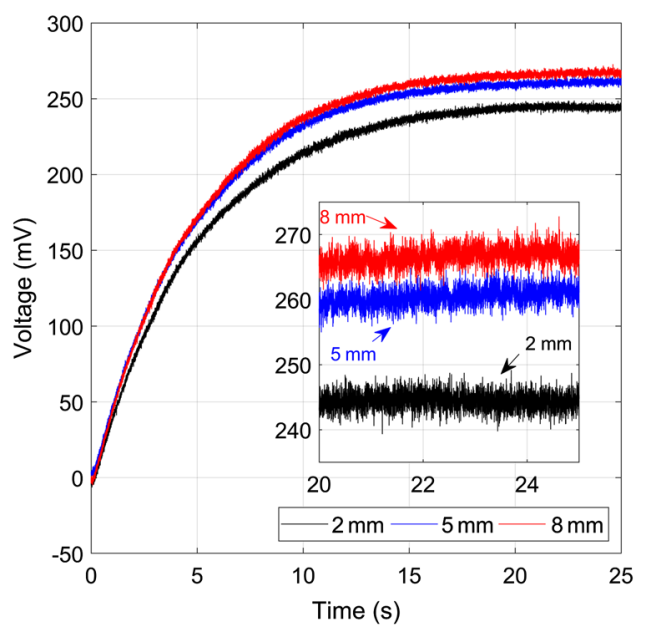

Fig. 5 Comparison of TEG open-circuit voltage responses to 808-nm, 25-W laser strikes with spot size diameters of 2, 5, and $8 \mathrm{~mm}$. The plotted voltage responses are low-pass filtered in postprocessing with a cut-off frequency of $1 \mathrm{~Hz}$ to reduce noise for ease of comparison. With fixed net incident power, increasing spot size results in slightly greater open-circuit voltages. 
trials had a noise rms level ranging from 4.7 to $6.2 \mathrm{mV}$. There was no discernible difference between noise levels preceding a laser strike and those recorded during a laser strike. In some trials, the noise level slightly increased, and in others, the noise level slightly decreased or remained the same. The $10 \%$ to $90 \%$ rise times for the open-circuit voltage response were also calculated for each trial, which indicated a correlation between higher power lasers and faster rise times. The rise times for the 1070-nm experiments were $9.3 \mathrm{~s}$ at $25 \mathrm{~W}$ and decreased to $6.58 \mathrm{~s}$ for the $100 \mathrm{~W}$. For the 25- and 50-W 1980-nm laser strikes, the rise times were 9.34 and $6.97 \mathrm{~s}$, respectively. The open-circuit voltage responses typically exceeded three times the prestrike rms level of the noise for that trial within $\sim 10$ to $\sim 300$ ms. Table 2 summarizes the observed noise levels and time required to exceed a voltage three times the rms noise levels.

There appears to be a weak correlation between the net power level of the laser strike and the time at which the open-circuit voltage responses exceeded the detection threshold of three times the rms noise level. For 1070-nm strikes with power ranging from 25 to $75 \mathrm{~W}$ and 1980-nm strikes with power ranging from 25 to $50 \mathrm{~W}$, increasing power corresponded with decreasing time for the response to exceed the detection threshold. However, the 1070-nm 100-W strike results were not consistent with this trend, and the $808-\mathrm{nm}$ strikes showed no correlation between incident laser power and the time to exceed the detection threshold. An example trace is shown in Fig. 6 for a 1070-nm 75-W laser strike in which the voltage first exceeds three times the prestrike rms level of the noise within $127 \mathrm{~ms}$.

TEG short-circuit current responses were also recorded to assess the potential of a TEGbased sensor to self-power notification circuitry. The short-circuit current responses to 808-nm

Table 2 Noise rms levels and time to exceed three times rms noise levels for selected trials.

\begin{tabular}{|c|c|c|c|c|c|c|c|c|c|}
\hline \multirow{2}{*}{$\frac{\lambda}{\text { Power (W) }}$} & \multicolumn{3}{|c|}{$808 \mathrm{~nm}$} & \multicolumn{4}{|c|}{$1070 \mathrm{~nm}$} & \multicolumn{2}{|c|}{$1980 \mathrm{~nm}$} \\
\hline & 15 & 25 & 35 & 25 & 50 & 75 & 100 & 25 & 50 \\
\hline Irradiance $\left(\mathrm{W} / \mathrm{cm}^{2}\right)$ & 29.8 & 49.7 & 69.6 & 49.7 & 99.5 & 149.2 & 198.9 & 49.7 & 99.5 \\
\hline Noise rms or $\sigma(\mathrm{mV})$ & 5.1 & 5.3 & 5.1 & 5.0 & 5.2 & 5.8 & 13.2 & 5.5 & 5.3 \\
\hline Time to exceed $3 \sigma$ noise $(\mathrm{ms})$ & 12.6 & 60.0 & 10.7 & 226.0 & 158.5 & 126.7 & 279.8 & 142.3 & 108.6 \\
\hline
\end{tabular}

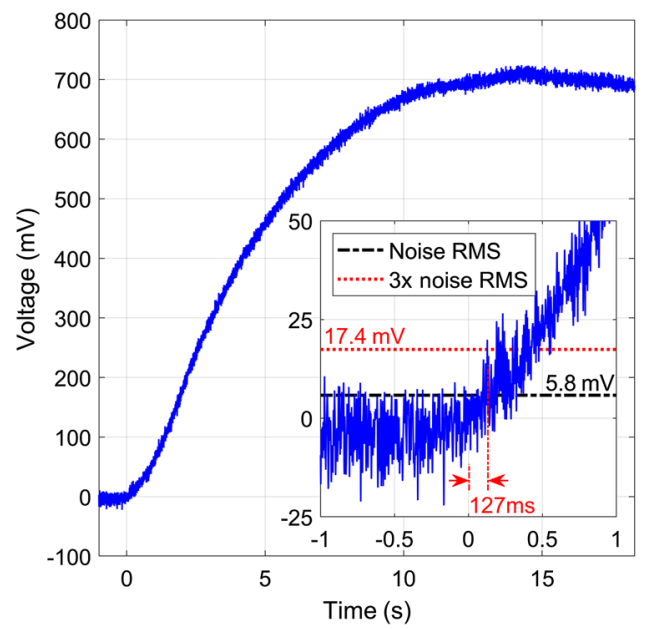

Fig. 6 The open-circuit voltage response of a TEG to a 1070-nm 75-W laser strike is plotted against time with the laser starting to strike the TEG at $t=0 \mathrm{~s}$. In the enlarged inset graph, the rms noise level, a chosen threshold level at three times the rms noise level, and the time from the start of the laser strike to the first open-circuit voltage data point exceeding the threshold level are annotated. The result is typical of the open-circuit voltage responses of the tested TEGs, which exceeded three times the rms noise levels recorded prior to the strike within time scales ranging from tens to a few hundred milliseconds. 

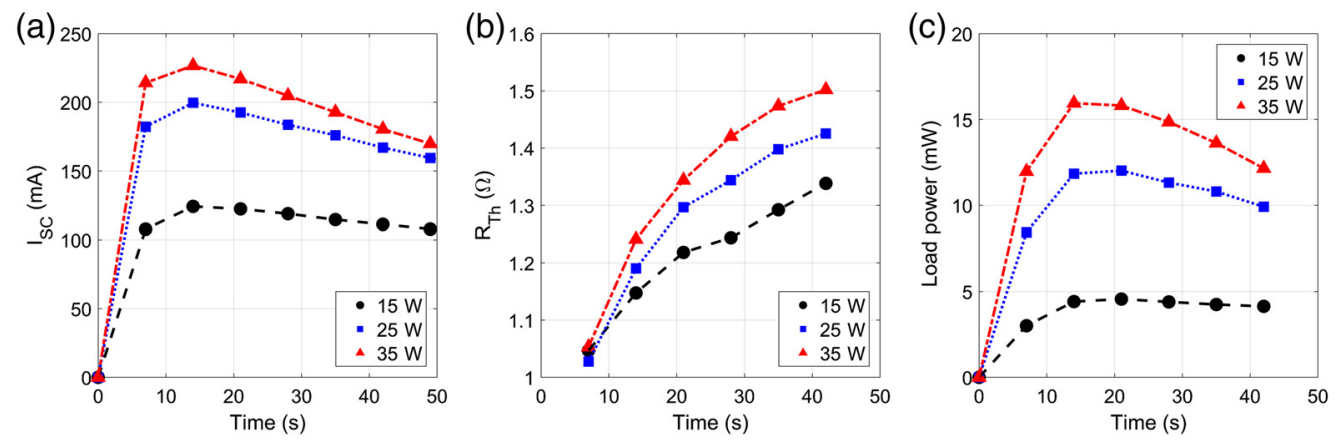

Fig. 7 (a) TEG short-circuit current $I_{\mathrm{SC}}$ responses to 808-nm laser strikes with spot size diameter of $15 \mathrm{~mm}$ and varying power levels, (b) calculated TEG Thévenin resistance $R_{\mathrm{Th}}$ from open-circuit voltage $V_{\mathrm{OC}}$ and from short-circuit current $I_{\mathrm{SC}}$ where $R_{\mathrm{Th}}=V_{\mathrm{OC}} / I_{\mathrm{SC}}$, and (c) estimated harvested power $P_{\text {Load }}$ to a fixed load with resistance $R_{\text {Load }}=1.28 \Omega$ where $P_{\text {Load }}=V_{\text {oc }} 2 R_{\text {Load }} /$ $\left(R_{\text {Th }}+R_{\text {Load }}\right)^{2}$.

laser strikes with an estimated spot size of 15-mm diameter and varying nominal input powers are shown in Fig. 7(a). The short-circuit current responses indicate $10 \%$ to $90 \%$ rise times of $\sim 6 \mathrm{~s}$, which are faster than the corresponding open-circuit voltage rise times of $\sim 10 \mathrm{~s}$. The shortcircuit current responses also show a more pronounced decline in the output response after reaching a peak level as compared to the open-circuit voltage response, particularly at high incident laser power levels. We postulate that the short-circuit current response is affected by increasing resistivity of the thermoelectric and conductive materials with increasing temperature, whereas this increased resistivity has limited effect on the open-circuit voltage magnitude. Both the shortcircuit current response and the open-circuit voltage response are likely affected by decreasing Seebeck coefficients at temperatures above approximately $150^{\circ} \mathrm{C}$.

By taking the ratio of open-circuit voltage to short-circuit current responses, an effective TEG Thévenin resistance $R_{\mathrm{Th}}$ can be calculated as shown in Fig. 7(b), with this resistance increasing with longer duration of irradiation. A hypothetical fixed load $R_{\text {Load }}$ connected to the TEG could be approximately matched to the TEG's Thévenin resistance $R_{\mathrm{Th}}$ by setting the load resistance $R_{\text {Load }}$ to the mean of $1.28 \Omega$ for the calculated effective Thévenin resistances of the various experimental data points. Figure 7(c) shows calculation results for potential peak harvested electrical power delivered to a 1.28- $\Omega$ fixed load from a TEG irradiated with an 808-nm laser strike, using a TEG circuit model with a voltage source $V_{\mathrm{OC}}$ and Thévenin resistance $R_{\mathrm{Th}}$, which both vary over the duration of the strike. Peak load power harvested is estimated to be 4.5 to $16 \mathrm{~mW}$ for nominal 808-nm laser powers of 15 to $35 \mathrm{~W}$.

Finally, when connected in series with other TEGs, there was no discernible difference in the voltage response compared to the experiments with only one TEG. In a scenario where all three TEGs were functional and the middle one was struck, the other two TEGs behaved like short circuits. In the experiments where one of the TEGs in series was replaced with a previously failed TEG, no voltage was detected across the series connection. Broken TEGs behaved like opencircuits, effectively eliminating the entire system's ability to detect a strike when connected in series. This result suggests that the TEGs tested tend to fail open when exposed to high temperatures resulting from high-energy laser strikes.

\subsection{Simulation Results}

The open-circuit voltage responses and average surface temperatures of time-dependent transient simulations were recorded for a wide range of simulation parameter sweeps. First, parameters for convective cooling coefficients and emissivity at the irradiation wavelength were adjusted to match IR camera experimental results for average surface temperatures. The emissivity of the aluminum oxide was the primary parameter to distinguish the wavelength of the lasers being emulated in simulation. Concurrently, the shape of the simulated transient surface temperature response was evaluated to tune the convective heat transfer coefficients on the front and back aluminum oxide substrates. The results of the simulations are compared to experimental data 
in Fig. 8. Reasonable agreement is obtained, although the simulated response at $808 \mathrm{~nm}$ could be further optimized for accuracy.

Based on simulation results that approximate the observed experimental responses, we estimate effective emissivities at 808, 1070, and $1980 \mathrm{~nm}$ for the material tested as $0.180,0.160$, and 0.375 , respectively. These emissivity estimates are comparable to measurements previously reported at these near-IR wavelengths. ${ }^{17}$ The tuned effective heat transfer coefficients for the 808-nm simulation were $h_{\text {front }}=h_{\text {back }}=15 \mathrm{~W} /\left(\mathrm{m}^{2} \mathrm{~K}\right)$, while the effective heat transfer coefficients for both the 1070- and 1980-nm simulations were $h_{\text {front }}=50 \mathrm{~W} /\left(\mathrm{m}^{2} \mathrm{~K}\right)$ and $h_{\text {back }}=0 \mathrm{~W} /\left(\mathrm{m}^{2} \mathrm{~K}\right)$. These estimates correspond well with the experimental conditions. For the 1070- and 1980-nm experiments, the TEG was mounted on a bracket that covered the rear side, and a low-speed fume extractor was positioned above the TEG. By contrast, the 808-nm experiments were conducted with the TEG front and back exposed and without a fume extractor. Therefore, the front-side convective heat transfer coefficient for the 808-nm laser experiments is expected to be less than those for the 1070- and 1980-nm experiments while the heat transfer coefficient for the rear side of the TEG in the 808-nm laser experiments could be expected to exceed those for the 1070- and 1980-nm experiments where the back of the TEG was not exposed.
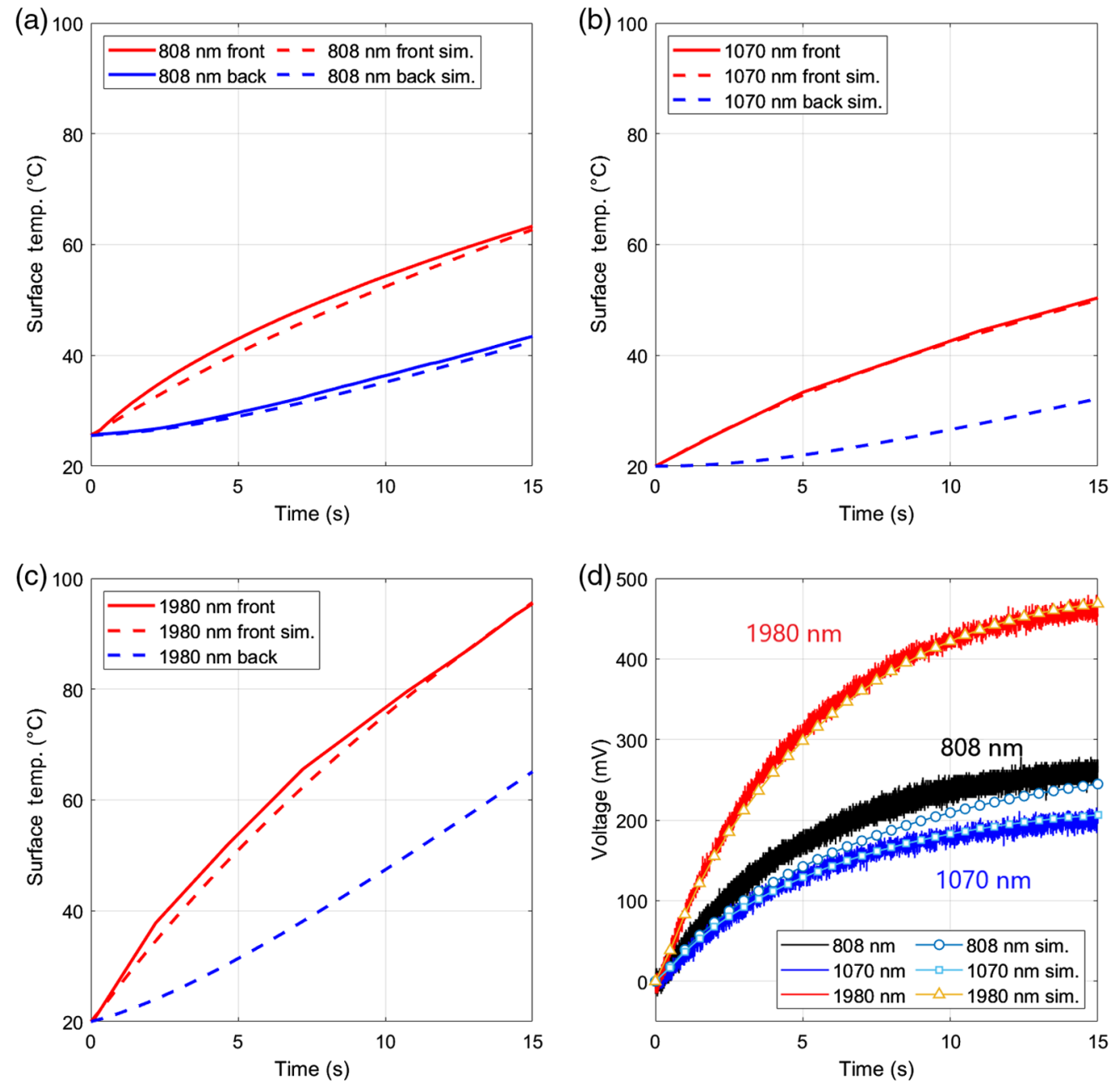

Fig. 8 Simulated average substrate surface temperatures with tuned simulation parameters compared to experimental results for laser strikes commencing at $t=0 \mathrm{~s}$ with nominal input laser powers of $25 \mathrm{~W}$ at (a) $808 \mathrm{~nm}\left[\varepsilon=0.180, h_{\text {front }}=h_{\text {back }}=15 \mathrm{~W} /\left(\mathrm{m}^{2} \mathrm{~K}\right)\right]$, (b) $1070 \mathrm{~nm}[\varepsilon=$ $\left.0.160, h_{\text {front }}=50 \mathrm{~W} /\left(\mathrm{m}^{2} \mathrm{~K}\right), h_{\text {back }}=0 \mathrm{~W} /\left(\mathrm{m}^{2} \mathrm{~K}\right)\right],(\mathrm{c}) 1980 \mathrm{~nm}\left[\varepsilon=0.375, h_{\text {front }}=50 \mathrm{~W} /\left(\mathrm{m}^{2} \mathrm{~K}\right)\right.$, $\left.h_{\text {back }}=0 \mathrm{~W} /\left(\mathrm{m}^{2} \mathrm{~K}\right)\right]$. Only the irradiated surface temperatures were recorded for the 1070- and 1980-nm experiments. (d) Simulated open-circuit voltage responses to the same laser strikes under the same simulated conditions compared to experimental results. 
The simulated open-circuit voltage responses were tuned by scaling the Seebeck coefficient via parameter $\beta$ after tuning the simulated thermal conditions to approximately match experimental surface temperature data. Good agreement in the simulated and experimental open-circuit voltage responses was obtained for nominal 25-W laser strikes at 808, 1070, and $1980 \mathrm{~nm}$ with Seebeck scaling coefficients of $\beta=1.10,1.06$, and 1.33, respectively. While the Seebeck coefficient is not considered to be a function of laser wavelength, the Seebeck coefficient is a function of temperature, which varies for both the simulation and experiments indirectly with wavelength via the wavelength-dependent emissivity of the TEG's aluminum oxide substrate material. The significant increase in tuned Seebeck scaling coefficient $\beta$ for the 1980-nm simulation relative to the 1070- and 808-nm simulations corresponds to higher surface temperatures $\left(\sim 100^{\circ} \mathrm{C}\right)$ observed for the corresponding experiment when compared to nominal $25-\mathrm{W}$ laser strike tests at other wavelengths $\left(\sim 60^{\circ} \mathrm{C}\right.$ to $\left.70^{\circ} \mathrm{C}\right)$. This result suggests that at least in the range of $60^{\circ} \mathrm{C}$ through $100^{\circ} \mathrm{C}$, the Seebeck coefficient of the TEGs tested increases with increasing temperature and increases more rapidly than the built-in COMSOL model assumes.

Simulation results were also used to validate the experimental conclusion that open-circuit voltage responses were more correlated with net power incident on the TEG than with specific irradiance levels of the laser strike. Figure 9(b) compares simulated open-circuit voltage responses for laser strikes of the same nominal incident power with differing spot sizes. The simulated results indicate a less than $1 \%$ increase in open-circuit voltage for laser strikes of the same integrated net incident power with peak irradiance levels varying by more than $400 \%$ for $1.25 \mathrm{~mm}<\sigma_{\text {spot }}<2.75 \mathrm{~mm}$. These simulated results contrast slightly with the experimental results, which showed a $\sim 2 \%$ increase in open-circuit voltage when increasing the nominal spot size from 5 to $8 \mathrm{~mm}$. Additionally, the simulated voltages increase at a faster time rate throughout the simulated laser strike duration due to imperfect thermal parameter fitting for the $808-\mathrm{nm}$ simulation. We attribute the small increase in open-circuit voltage with increasing spot size

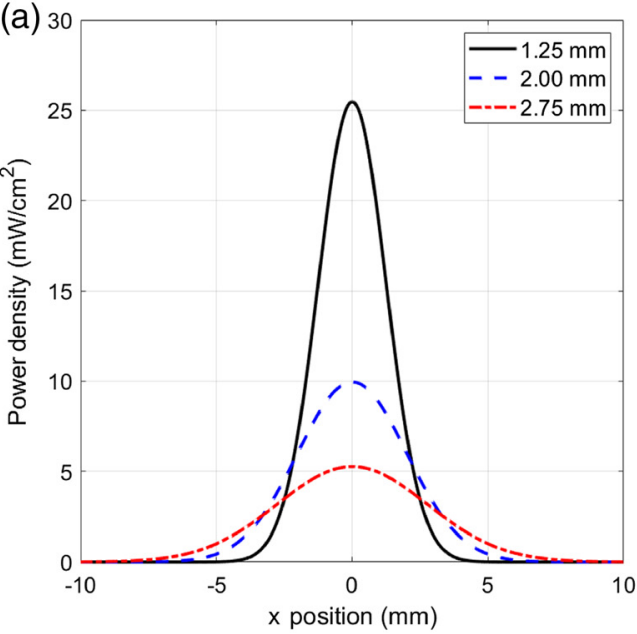

(c)

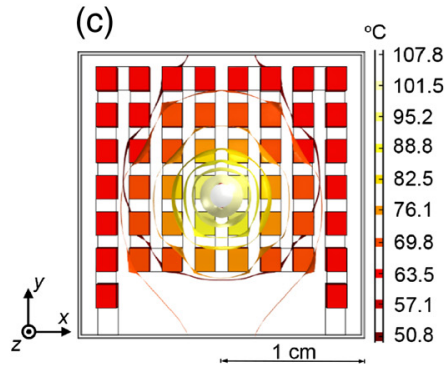

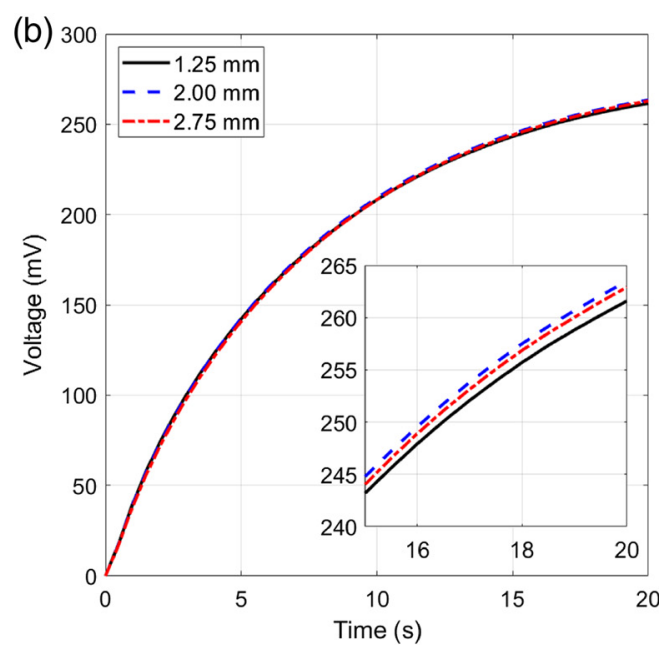

(d)

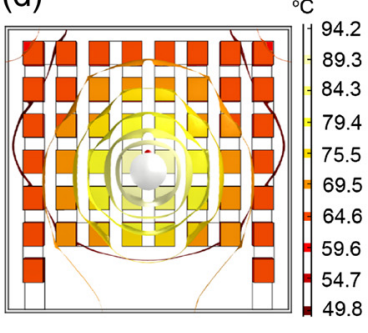

(e)

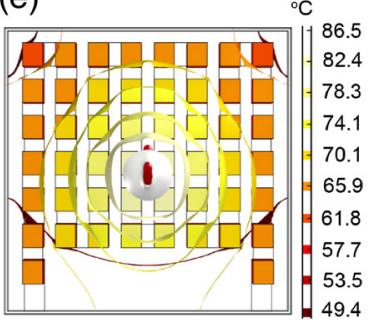

Fig. 9 (a) Simulated Gaussian profiles for various standard deviations $\sigma_{\text {spot }}$ taken along the $y=0$ line centered on the simulated TEG and positioned along its front surface. (b) Open-circuit voltage responses with varying spot size standard deviations. Simulated isothermal contours of the irradiated TEGs $20 \mathrm{~s}$ after the start of the simulated 808-nm laser strike with spot size standard deviations of $1.25,2$, and $2.75 \mathrm{~mm}$ are shown in (c), (d), and (e), respectively. 
observed in both simulated and experimental results to the development of thermal gradients across more thermoelectric couples with larger spot sizes. While the temperatures are greater in the smaller spot size cases, fewer thermoelectric couples are affected.

More focused laser beams of the same net power did result in higher peak surface temperatures both in experiment and in simulation. Simulated peak surface temperatures ranged from $107.8^{\circ} \mathrm{C}$ at $\sigma_{\text {spot }}=1.25 \mathrm{~mm}$ to $86.5^{\circ} \mathrm{C}$ at $\sigma_{\text {spot }}=2.75 \mathrm{~mm}$. These results suggest that the primary consideration in the magnitude of the open-circuit voltage response is the difference in average temperatures between the front (irradiated) and back (nonirradiated) substrates of the TEG. Average simulated surface temperatures did not vary significantly with variations in spot size in the simulations conducted.

\section{Discussion}

The basic functionality of a TEG as a passive sensor for near-IR laser irradiation is verified by experiment and simulation. Both open-circuit voltage and thermoelectrically generated power could serve as sensor output signals. The TEG response is related to the thermal gradient through the thickness of the TEG. The thermal gradient is limited by conduction through the TEG, resulting in a limit to the sensor output response to a strike of a given power even as TEG surface temperatures continue to climb. TEG open-circuit voltage response maximum increases with increasing net incident power on the TEG surface. The maximum in the open-circuit voltage response is more closely correlated with net incident power on the TEG surface than with specific irradiance. For fixed-duration continuous-wave laser strikes of wavelengths 808,1070 , and $1980 \mathrm{~nm}$, the tested TEGs exhibited an open-circuit voltage (at an arbitrary $t=4 \mathrm{~s}$ ) to nominal incident laser power ratios of $6,4.9$, and $11.1 \mathrm{mV} / \mathrm{W}$ with linearity most likely limited by variation in the Seebeck coefficient with temperature.

A fundamental limitation of the TEG as a high-energy laser strike sensor is that its performance degrades with the primary variable it seeks to measure. The gradual degradation of the sensor performance at increasing temperatures is evidenced by the apparent decrease in Seebeck coefficient and conductivity of the electrical elements in the experimental results. Reducing temperatures via optically reflective surfaces or thermal management is possible but requires close optimization since such techniques shield the sensor from the measured variable. Simulations show that increased emissivity of the TEG substrate at wavelengths of interest can increase the open-circuit voltage response, but increased sensitivity via heat absorption risks further damage to the sensor and possibly to nearby components. Therefore, the combination of surface materials of a sensor-integrated frame needs to be evaluated carefully.

Other limitations of the tested TEGs as laser strike sensors include significant sensor thermal mass, which increases the sensor response time to dynamic irradiation conditions. However, the open-circuit voltage responses still typically exceeded three standard deviations of the typical noise voltage within less than $300 \mathrm{~ms}$ of the start of a laser strike for the experiments conducted. Resistive thermal noise is the probable major noise contributor. Using the same measurement settings used to observe the TEG open-circuit voltage responses and postprocessing with a highpass filter function in MATLAB ${ }^{\circledR}$, the typical rms noise levels of the tested TEGs were calculated to be $\sim 5.4 \mathrm{mV}$ over a Nyquist bandwidth of $\sim 5 \mathrm{kHz}$. Noise was observed to increase by less than 5\% when comparing rms noise voltages before a laser strike and during the laser strike in most experiments analyzed. The low bandwidth of the signal response $(<1 \mathrm{~Hz})$ allows most noise to be effectively filtered.

A unique advantage of a TEG as a high-energy laser strike sensor is that a TEG has potential to be utilized as a self-powered passive sensor. The power generated by TEGs when irradiated enables an array of individual TEGs to cover the large surface area of a military vehicle, vessel, or aircraft without the interdependency of a series circuit arrangement, the loss in positional resolution of a parallel arrangement, or the overhead of active interrogation of each individual sensor. Irradiated TEGs could generate sufficient power to operate sensor node circuitry associated with each of those TEGs only after being irradiated, thereby reducing power consumption and minimizing possible disruption of stealth features until those stealth features are already compromised. Assuming a closely matched load, estimated ratios of maximum thermoelectric 
power per nominal incident laser power for the TEGs tested in this study are 267 to $457 \mu \mathrm{W} / \mathrm{W}$ for 808-nm laser strikes with nominal powers from 15 to $35 \mathrm{~W}$. The results presented here suggest additional testing is warranted to characterize variability in the power harvesting capability of TEGs deployed as high-energy laser detectors.

Future work to explore TEG arrangements as self-powering passive high-energy laser strike sensors will include flexible thin-film designs to permit low-profile integration into composite surfaces and reduce response times by reducing thermal mass. The use of thermoelectric power during a laser strike to power sensor processing electronics also deserves further study. Detectivity can be optimized by selecting thermoelectric materials suitable for elevated temperatures and TEG substrates that are highly absorptive at the expected incident laser wavelengths. In a practical deployment, detectivity would also need to be balanced by survivability concerns. Various integrations of TEG sensors into composites suitable for vehicles or other expected high-energy laser strike targets can be studied to optimize sensitivity and robustness in the expected operating environments.

\section{Appendix A: Simulation Parameters}

Table 3 summarizes relevant simulation dimensions and parameters. Parameter descriptions and determination methods are described in Sec. 2.2.

Table 3 Simulation parameters.

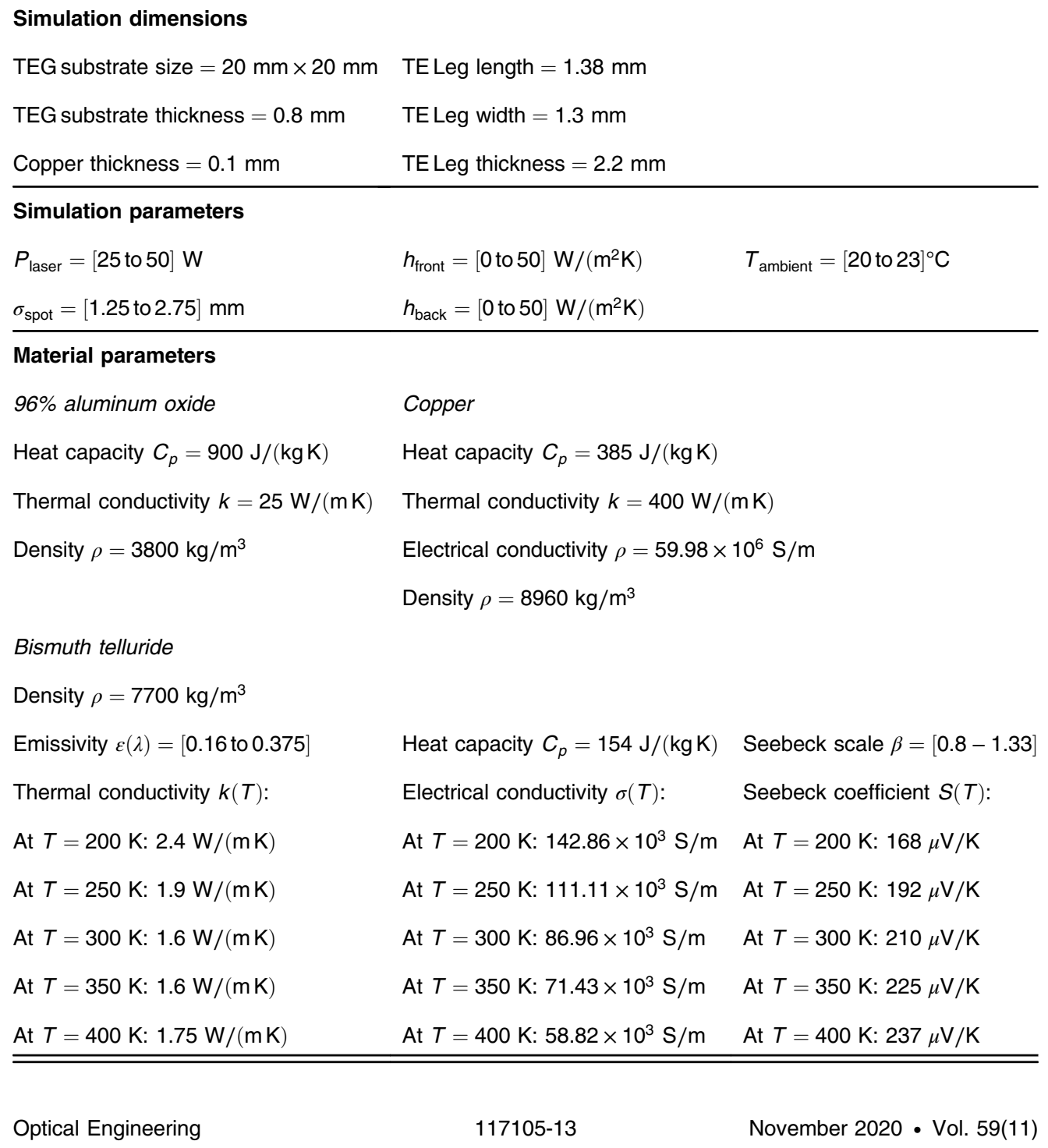




\section{Acknowledgments}

We would like to thank the Naval Surface Warfare Center (NSWC) in Crane, Indiana, for providing their equipment, laboratories, and expertise for many of the initial experiments. We would also like to thank the Office of Naval Research (ONR) and Directed Energy Joint Transition Office (DE-JTO) for providing funding for our research.

\section{References}

1. D. R. Jungwirth, "Laser detection and warning system," US 9134174 B2, United States Patent and Trademark Office (2013).

2. G. Muller, "Laser-warning device for military vehicles," US 4721852 A, United States Patent and Trademark Office (1984).

3. D. M. Mechtel et al., "Radio frequency switching network: a technique for infrared sensing," Opt. Eng. 55(10), 107106 (2016).

4. D. Mechtel et al., "Apparatus and method for detection of electromagnetic radiation using optical switches in an RF network," US 9500522 B2, United States Patent and Trademark Office (2016).

5. A. Kong et al., "Rapid detection of high energy laser strikes using distributed optical fiber sensors," in 21st Annu. Directed Energy Sci. and Technol. Symp., Directed Energy Professional Society (2019).

6. M. J. Ross et al., "High temperature effects during high energy laser strikes on embedded fiber Bragg grating sensors," Sensors 19, 1432 (2019).

7. R. B. Jenkins, P. Joyce, and D. Mechtel, "Localized temperature variations in laser-irradiated composites with embedded fiber Bragg grating sensors," Sensors 17, 251 (2017).

8. G. S. Glaesemann, "WP8002: optical fiber mechanical reliability-review of research at Corning's Optical Fiber Strength Laboratory," Dow Corning (2017).

9. Fiberoptics Technology Incorporated, "Operating temperature," https://www.fiberopticstech .com/technical/operating-temperature (5 May 2020).

10. R. B. Jenkins et al., "Discerning localized thermal heating from mechanical strain using an embedded distributed optical fiber sensor network," Sensors 20, 2583 (2020).

11. Accuratus, "Aluminum oxide, $\mathrm{Al}_{2} \mathrm{O}_{3}$ ceramic properties," 2013, https://accuratus.com/ alumox.html (16 December 2019).

12. National Center for Biotechnology Information, "Aluminum oxide, CID = 9989226," PubChem Database, https://pubchem.ncbi.nlm.nih.gov/compound/Aluminum-oxide (16 December 2019).

13. CUI Inc., "Peltier module," CP602040 Datasheet (2018).

14. L. M. Goncalves et al., "Flexible thin-film planar Peltier microcooler," in 25th Int. Conf. Thermoelectr., pp. 327-331 (2006).

15. N. Sato and M. Takeda, "Fabrication and evaluation of a flexible thermoelectric device using metal thin films," in 24th Int. Conf. Thermoelectr., pp. 175-178 (2005).

16. J. Merkel et al., "High energy laser detection through thermoelectric generators," Proc. SPIE 11382, 1138204 (2020).

17. MatWeb, "Overview-Alumina $96 \% \mathrm{Al}_{2} \mathrm{O}_{3}$," http://www.matweb.com/search/datasheet print.aspx?matguid=204094cb0fd34c099b8a52c9c7df1d5c (10 March 2020).

18. C. H. Liebert, "Spectral emittance of aluminum oxide and zinc oxide on opaque substrates," NASA Technical Note D-33115 (1965).

19. G. C. Dannangoda et al., "Transition of $p$ - to $n$-type conductivity in mechanically activated bismuth telluride," J. Electron. Mater. 47(10), 5800-5809 (2018).

Joseph Merkel is a recent graduate of the United States Naval Academy (USNA), where he obtained a bachelor of science in electrical engineering. He currently serves as an officer in the U.S. Navy and is pursuing a career as a pilot.

Steven C. Yee received his BS degree in electrical engineering from the USNA and his MS degree in electrical engineering from Massachusetts Institute of Technology. He is a lieutenant 
in the U.S. Navy's Civil Engineer Corps assigned as an electrical engineering instructor at the USNA since 2018. His current research interests include photothermal sensing and actuation, microrobotics, and additively manufactured radio-frequency (rf) components.

Charles Nelson is an associate professor of electrical and computer engineering at the USNA. He received his BS degree in physics from the USNA (1996), an MEM degree from Old Dominion University in 2003, and MS and PhD degrees in electrical and computer engineering from Johns Hopkins University in 2004 and 2013, respectively. His research areas primarily focus on free space and underwater laser beam propagation, directed energy detection, and atmospheric optical turbulence characterization.

Brian Jenkins received his BS and MS degrees in electrical engineering from The Ohio State University, and his $\mathrm{PhD}$ from the University of Colorado at Boulder. He worked for six years at IBM in processor development and has taught electrical engineering at the USNA since 1996. His research interests include optical fiber sensing and materials for structural health monitoring, as well as photonic networks, nonlinear fiber optics, and optical communications.

Hatem ElBidweihy is an associate professor of electrical and computer engineering at the USNA. He received his BS and MS degrees from Cairo University, Egypt, in 2009 and 2011, and $\mathrm{PhD}$ in electrical engineering from the George Washington University in 2014. He specializes in characterizing and modeling magnetic materials and designing magnetic circuits for sensors and actuators.

Peter J. Joyce received his BS degree in engineering mechanics from the University of Illinois, Urbana-Champaign. He received his $\mathrm{PhD}$ in materials science and engineering from the University of Texas at Austin. He has been teaching in the Mechanical Engineering Department at the USNA since 1999. His research areas include experimental mechanics, composite materials, multifunctional materials, additive manufacturing, and high-energy laser/materials interactions.

Cody J. Brownell is a professor in the Mechanical Engineering Department at the USNA in Annapolis, Maryland. He holds a BSE degree in mechanical engineering from Duke University, and MS and PhD degrees in mechanical engineering from Johns Hopkins University. His research interests involve lasers and fluid dynamics, including atmospheric optical turbulence, high-energy propagation through water drops, and the use of novel laser-based imaging techniques to measure gas-phase turbulent flows.

Deborah Mechtel received her BS degree in mechanical engineering from the University of Virginia and her MS and PhD degrees in electrical engineering from Johns Hopkins University. She has taught electrical engineering at the USNA since 1994. Her current research interests include optical sensors and RF photonic sensors for high-energy applications and instrumentation and material characterization for RF applications. 